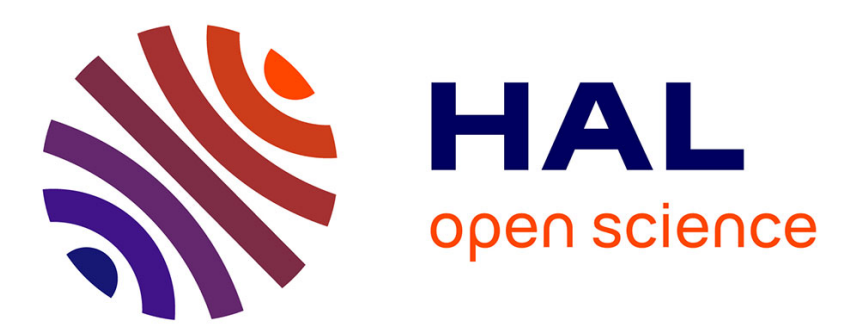

\title{
A generalization of the local graduai deformation method using domain parameterization
}

Benjamin Marteau, Didier-Yu Ding, Laurent Dumas

\section{To cite this version:}

Benjamin Marteau, Didier-Yu Ding, Laurent Dumas. A generalization of the local graduai deformation method using domain parameterization. Computers \& Geosciences, 2014, 7, pp.233-243. 10.1016/j.cageo.2014.08.004 . hal-01086000

\section{HAL Id: hal-01086000 https://hal-ifp.archives-ouvertes.fr/hal-01086000}

Submitted on 21 Nov 2014

HAL is a multi-disciplinary open access archive for the deposit and dissemination of scientific research documents, whether they are published or not. The documents may come from teaching and research institutions in France or abroad, or from public or private research centers.
L'archive ouverte pluridisciplinaire HAL, est destinée au dépôt et à la diffusion de documents scientifiques de niveau recherche, publiés ou non, émanant des établissements d'enseignement et de recherche français ou étrangers, des laboratoires publics ou privés. 


\title{
A generalization of the local gradual deformation method using domain parameterization
}

\author{
Benjamin Marteau ${ }^{\mathrm{a}, *}$, Didier-Yu Ding ${ }^{\mathrm{a}}$, Laurent Dumas ${ }^{\mathrm{b}}$ \\ a IFP énergies nouvelles avenue Bois Préau, Rueil-Malmaison, France \\ ${ }^{b}$ Université de Versailles Saint-Quentin, avenue de Paris, Versailles, France
}

\begin{abstract}
Reservoir model needs to be constrained by various data, including dynamic production data. Reservoir heterogeneities are usually described using geostatistical approaches. Constraining geological/geostatistical model realizations by dynamic data is generally performed through history matching, which is a complex inversion process and requires a parameterization of the geostatistical realizations for model updating. However, the parameterization techniques are still not very efficient and need to be improved.

In recent years, the local gradual deformation method has been widely used to parameterize geostatistical realizations. The domain deformation technique has also been developed to improve the history matching efficiency. Both methods can smoothly modify model realizations while conserving spatial geostatistical properties. The first one consists in locally combining two or more realizations while the second one allows the optimization process to change the model realization via the variation of the shape of geometrical domains. In this paper, we generalize the local gradual deformation method by adding the possibility to change the geometry of local zones through the domain deformation. This generalization provides a greater flexibility in the definition of the local domains for the local gradual deformation method. In addition, we propose a new way to initialize the realization which guaranties a good initial point for the optimization and potentially improves the efficiency of history matching.

Keywords: History matching, Geostatistical realizations, Parameterization, Gradual deformation, Domain deformation
\end{abstract}

\section{Introduction}

A reservoir model is built based on both static and dynamic data. Static data represent the data obtained from experiments carried out on cores extracted from wells or measurements of well logs such as porosity or permeability. Dynamic data are generally the well production data such as well pressure, oil rate, etc.. The s integration of dynamic data in the reservoir model is generally performed through history matching.

\footnotetext{
*Benjamin Marteau

Email address: benjamin.marteau@ifpen.fr (Benjamin Marteau )
} 
Reservoir heterogeneities are described using geological/geostatistical approach. The uncertainty of a model realization is linked to the geological scheme, to the sedimentary concept, to the nature of the reservoir rocks, their extent, and to their properties. Ignoring the uncertainties in the reservoir lithology would lead to underestimating the complexity of the reservoir between wells, resulting in over or under-estimating the connected reservoir pore volumes. Integration of dynamic data to constrain the geostatistical realization can reduce greatly model uncertainties. Although reservoir heterogeneities are commonly generated using geostatistical models, random realizations cannot generally match observed dynamic data. To constrain model realizations to reproduce measured dynamic data, an optimization procedure may be applied in an attempt to minimize an objective function. Such history matching methods require a parameterization of 15 the geostatistical model to allow the updating of an initial model realization.

To parameterize the geostatistical model, several methods were introduced. For example, the pilot point method (Marsily et al., 1984), the gradual deformation method (Roggero \& Hu, 1998), the domain deformation method (Ding \& Roggero, 2010) and the probability perturbation method (Hoffman \& Caers, 2003) were all proposed to continuously deform the models. All these methods allow the modification of a geostatistical realization by preserving its spatial variability.

In recent years, the local gradual deformation method has been increasingly used (Roggero et al., 2007, Al-akhdar et al., 2012). In that method, the deformation zones are fixed and cannot be changed during history matching. If these zones are not suitably defined, it is difficult to decrease the objective function for history matching and to find an optimal realization. The choice of deformation zones is a critical point for the successful history matching of geostatistical realizations. In this paper, we propose a generalization of the local gradual deformation method, which can optimize the deformation zones through the domain deformation technique during a history matching process. This method allows the gradual deformation in varying domains and find more efficiently an optimal geostatistical realization.

Another issue in history matching is the selection of the initial model realization for the local gradual deformation. The batchwork method, which combines locally different realizations according to the matching results on the wells (Reis et al., 2000), is sometimes used to define an initial model. But this method does not always work well, and sometimes gives very bad results. Using the domain deformation technique alone may provide a suitable approach to define a convenient initial model.

In this paper, we will first briefly review the gradual deformation and the domain deformation methods, then present a generalization of the gradual deformation method by combining with the domain deformation technique to modify the shapes and sizes of the domains. We will also show how to get a convenient initial model for history matching by using the domain deformation technique. Examples are presented to show some promising results with the new technique. 


\section{A generalized local gradual deformation technique}

\subsection{Gradual deformation method}

Some geostatistical methods such as the Fast Fourier Transform Moving Average (FFT-MA) (Le Ravalec et al., 2000) allow us to uncouple uncorrelated random realizations from structured information (mean, variance, correlation length, etc). With such methods, a model realization $M$ is linked to a standard Gaussian white noise $Z$ by an operator $G$ :

$$
M=G(Z)
$$

The gradual deformation method (Gervais et al., 2007, $\mathrm{Hu}, 2002$, Roggero \& $\mathrm{Hu}$, 1998) consists in combining two or more Gaussian white noises to modify the model realization. More precisely, it uses the fact that if $\left(Z_{0}, \ldots, Z_{N}\right)$ are $N+1$ independent standard Gaussian white noises and $\left(a_{0}, \ldots, a_{N}\right)$ are $N+1$ real numbers such that $\sum_{i} a_{i}^{2}=1$, then $Z=\sum_{i} a_{i} Z_{i}$ is still a standard Gaussian white noise. Then, if the $a_{i}$ depend on a set of parameters $\rho=\left(\rho^{1}, \ldots, \rho^{N}\right)$ that guaranties that for all $\rho, \sum_{i} a_{i}^{2}(\rho)=1$, we can generate new model realizations for all $\rho$. Moreover, a continuous variation of the set of parameters $\rho$ gives a continuous variation of the spatial properties of the model realization as illustrated in Fig.1. For example,
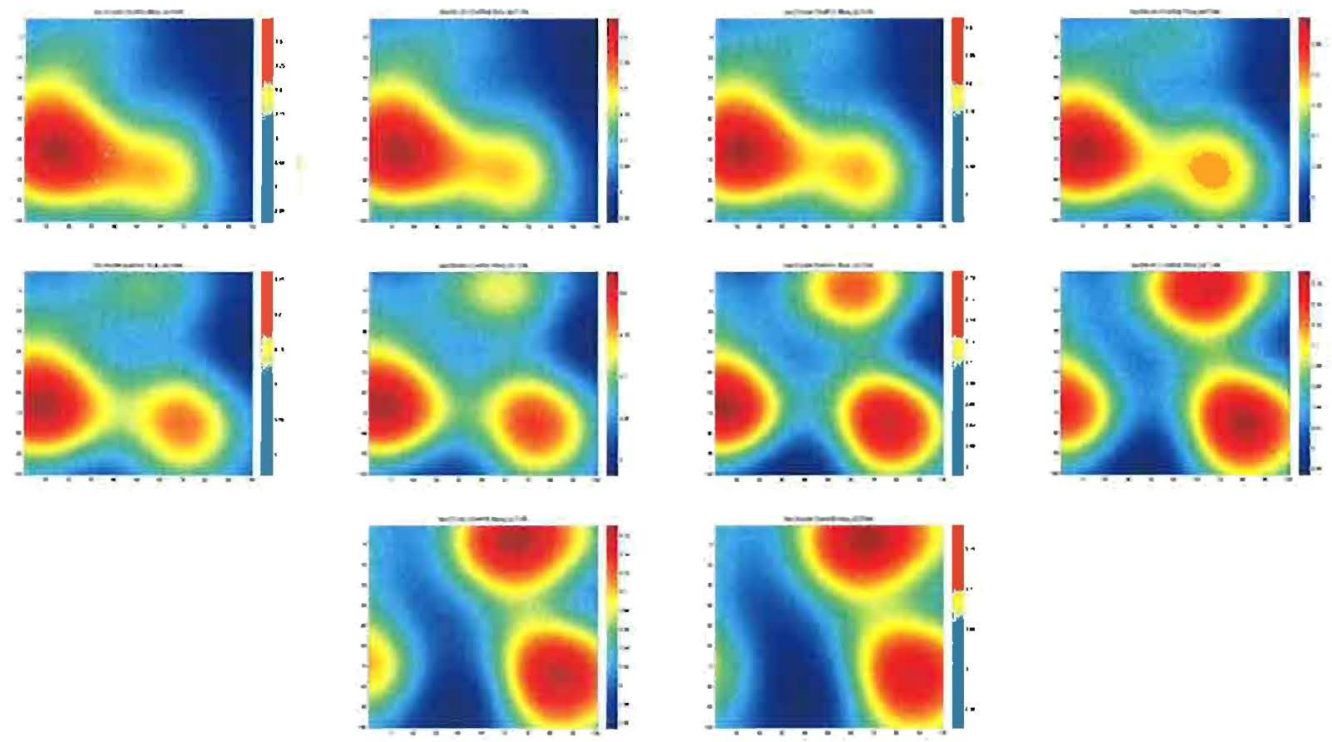

Figure 1: Model variation with the variation of a local gradual deformation parameter $\rho^{1}$

if we want to combine two independent Gaussian white noises $Z_{0}$ and $Z_{1}$, we can introduce a parameter $\rho^{1}$ and choose $Z\left(\rho^{1}\right)$ such as:

$$
Z\left(\rho^{1}\right)=\cos \left(\rho^{1}\right) Z_{0}+\sin \left(\rho^{1}\right) Z_{1}
$$


To combine $N+1$ Gaussian white noises $\left(Z_{0}, \ldots, Z_{N}\right)$, we introduce $N$ parameters $\rho=\left(\rho^{1}, \ldots, \rho^{N}\right)$. The gradual deformation is then given by:

$$
Z=\prod_{i=1}^{N} \cos \left(\rho^{i}\right) Z_{0}+\sum_{i=1}^{N-1} \sin \left(\rho^{i}\right) \prod_{k=i+1}^{N} \cos \left(\rho^{k}\right) Z_{i}+\sin \left(\rho^{N}\right) Z_{N}
$$

For local gradual deformation, we group the model grid cells in zones and locally combine several Gaussian white noises inside these zones. For example: if the model is divided into 2 zones and 3 standard Gaussian white noises are available:

$$
Z_{0}=\left[\begin{array}{c}
Z_{0, \text { zone }_{1}} \\
Z_{0, \text { zone }_{2}}
\end{array}\right], Z_{1}=\left[\begin{array}{c}
Z_{1, \text { zone }_{1}} \\
Z_{1, \text { zone }_{2}}
\end{array}\right] \text { and } Z_{2}=\left[\begin{array}{c}
Z_{2, \text { zone }_{1}} \\
Z_{2, \text { zone }_{2}}
\end{array}\right]
$$

we can define $Z(\rho)$ by :

$$
Z(\rho)=\left[\begin{array}{c}
\cos \left(\rho^{1}\right) Z_{0, z o n e_{1}}+\sin \left(\rho^{1}\right) Z_{1, z o n e_{1}} \\
\cos \left(\rho^{2}\right) Z_{0, z o n e_{2}}+\sin \left(\rho^{2}\right) Z_{2, z o n e_{2}}
\end{array}\right]
$$

with $\rho=\left(\rho^{1}, \rho^{2}\right) . Z(\rho)$ is still a standard Gaussian white noise and can thus still legitimately be used to generate a model realization through the operator $G$. In this case, $Z_{0}$ and $Z_{1}$ are combined in the domain zone $e_{1}$ and $Z_{0}$ and $Z_{2}$ are combined in the domain $z o n e_{2}$. This approach allows us to independently modify

as realizations in several regions of the model.

\subsection{Domains deformation method}

The domain deformation method (Ding \& Roggero, 2010) has some similarities to the local gradual deformation method. The model is divided into different zones (not necessarily delimited by grid cells) and a standard Gaussian white noise is restrained to each zone. The geostatistical model realization is then modified by deforming the shapes and sizes of the zones. Fig.2 shows an example of a reservoir model divided into two domains $R_{1}$ and $R_{2}$. We build the model realization with a standard Gaussian white noise associated to $Z_{1}$ inside $R_{1}$ and to $Z_{2}$ inside $R_{2}$. However, the random value is not clearly defined on grid cells that are partially on several domains. Let's consider the grid cell $X$ of the Fig. 2 which is not entirely inside any domain. To ensure the model continuity, we choose for this grid cell a combination of the two Gaussian white noises $Z_{1}$ and $Z_{2}$ as follows:

$$
Z_{X}=a_{1} Z_{1, X}+a_{2} Z_{2, X}
$$

where $a_{1}$ and $a_{2}$ depend on the shape and the size of the domains, which can be parameterized. As for the local gradual deformation method, $Z$ is a standard Gaussian white noise if $a_{1}^{2}+a_{2}^{2}=1$. We can choose, for example, $a_{i}$ proportional to the proportion of the grid cell inside the domain $i$. 


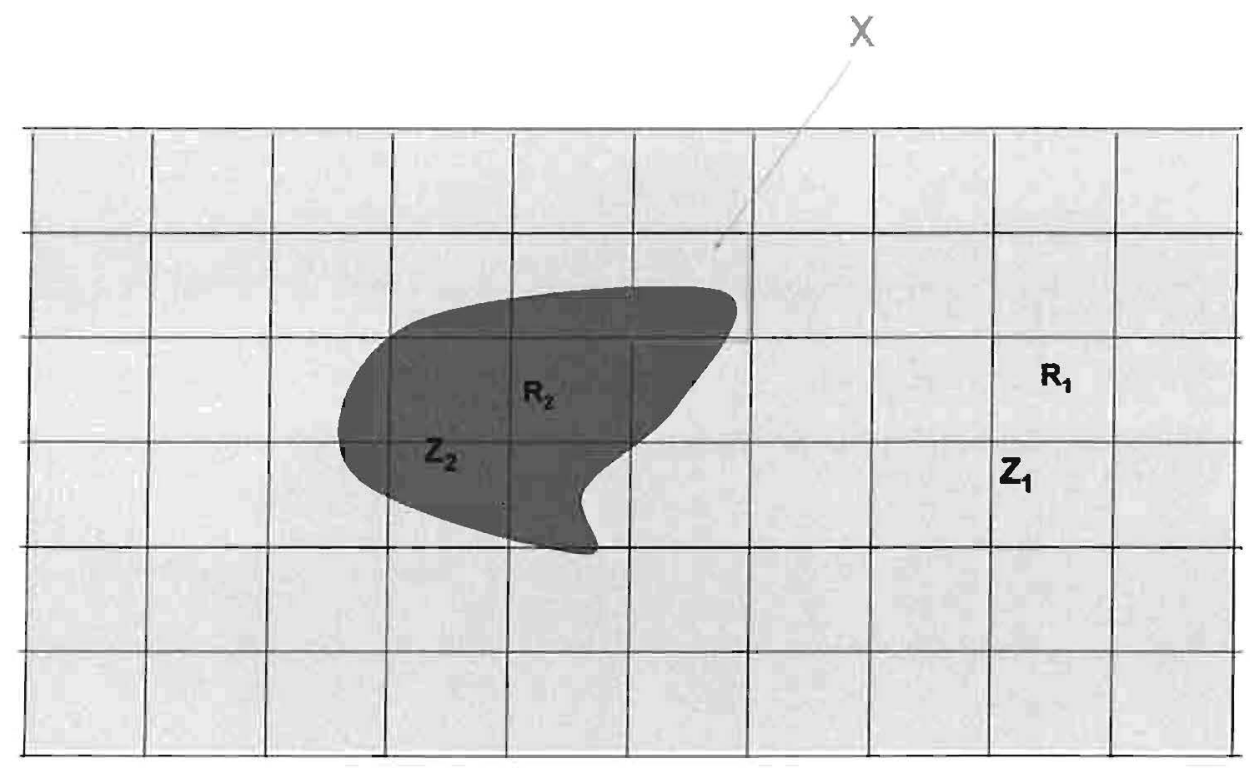

Figure 2: Reservoir model divided into 2 zones $R_{1}$ and $R_{2}$

The new model realization therefore depends on the parameters which define the shapes and sizes of the domains. In general, to limit the number of parameters, we choose simple shapes for the domains that depend only on a small number of parameters. For example, choosing circles with fixed centers allows us to determine each domain with only one parameter: their radius.

This method can be extended to the case of $M$ domains to deform with $N+1$ Gaussian white noises. Let $t_{i}=\left(t^{1}, \ldots, t^{q}\right)$ be the set of parameters determining the shape of the domain $R_{i}$ and $t=\left(t_{1}, \ldots, t_{M}\right)$ contain all the domain parameters, we can combine the Gaussian white noises:

$$
Z(X, t)=\sum_{i=0}^{M} a_{i}\left(X, t_{i}\right) Z_{J(i)}(X)
$$

where $a_{i}\left(X, t_{i}\right)$ depends on the shape of the domain $R_{i}, J(i) \in[0, \ldots, N]$ is the index of the Gaussian white noise associated to the domain $R_{i}$ and $J(0)=0$. The new standard Gaussian white noise is parameterized with $t$.

so The advantage of this parameterization technique compared to the local gradual deformation is that it is not very dependent on the initial domain selections, since their shapes and sizes can be modified. In fact, a bad definition of the zones in the local gradual deformation method could greatly deteriorate the potential diminution of the objective function. This is well illustrated in Fig.3 which was presented by Ding \& Roggero (2010) to compare the potential of the domain deformation method and the gradual deformation 
os method on a history matching problem. In this figure, the pink curve presents the variations of the objective function with the size of the domains, while the blue curve presents the optimal results using the local gradual deformation technique with fixed domains sizes. In this particular case, when the domain size (radius) is fixed, the contribution of the local gradual deformation is relatively limited while changing the domain size can rapidely reduce the objective function. This example shows that in certain cases, choosing well suited domains can be critical to the performance of the gradual deformation method.

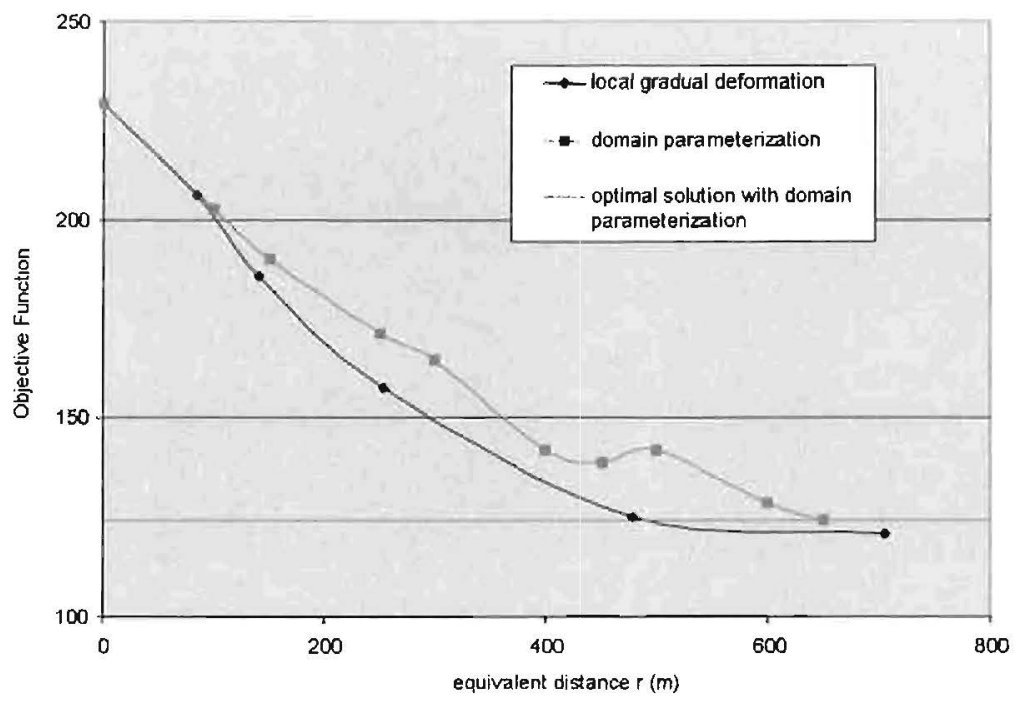

Figure 3: Comparison between the domain deformation method and the local gradual deformation method on a synthetic case

\subsection{Generalization of the gradual deformation method}

\subsubsection{Another way to write the domain deformation technique}

To be able to combine the gradual deformation and the domain deformation methods, we write them in a similar form. We present here a variant of the domain deformation method. Let's start by a simple case with only one zone and two model realizations.

One zone and two standard Gaussian white noises.

Let's Consider one zone $R_{1}$ parameterized by the set of parameters $t_{1}=\left(t^{1}, \ldots, t^{q}\right)$ and two independent model realizations associated to two Gaussian white noises $Z_{0}$ and $Z_{1}$. For a given grid cell $X$, we define $V_{1}\left(X, t_{1}\right)$ by the volume of the domain $R_{1}$ inside $X$ and $V_{0}\left(X, t_{1}\right)=\operatorname{vol}\left(X-R_{1}\right)$ its complementary. Let $\alpha_{1}$ be:

$$
\alpha_{1}\left(X, t_{1}\right)=\frac{V_{1}\left(X, t_{1}\right)}{V_{1}\left(X, t_{1}\right)+V_{0}\left(X, t_{1}\right)}
$$

A new gaussian white noise $Z(X)$ can be built with:

$$
Z(X)=\cos \left(\frac{\pi}{2} \alpha_{1}\right) Z_{0}(X)+\sin \left(\frac{\pi}{2} \alpha_{1}\right) Z_{1}(X)
$$


This Gaussian white noise associates $Z_{1}$ to the inside of the domain $R_{1}$ and $Z_{0}$ to the outside. It is a combination of the two on the grid cells partially occupied by the zone. The new model realization $G(Z)$ is dependent on the shape and size of $R_{1}$ which is parameterized with $t_{1}$.

Generalization: $M$ zones and $M+1$ Gaussian white noises.

We can generalize the above formula to the case of $M$ zones $\left(R_{1}, \ldots, R_{M}\right)$. If we have at our disposal $M+1$ Gaussian white noises $\left(Z_{0}, \ldots, Z_{M}\right)$ and a set $t=\left(t_{1}, \ldots, t_{M}\right)$ that parameterizes the shapes of all the zones, we can associate one model realization to each zone and the last one to the outside of every zone. As in the previous example, for a given grid cell $X$, we define $V_{i}\left(X, t_{i}\right)=\operatorname{vol}\left(X \cap R_{i}\right), V_{0}(X, t)=\operatorname{vol}\left(X-\cup_{j} R_{j}\right)$ and $\alpha_{i}$ :

$$
\alpha_{i}(X, t)=\frac{V_{i}\left(X, t_{i}\right)}{\sum_{j=0}^{M} V_{j}\left(X, t_{j}\right)}
$$

For the sake of simplicity, we decided to associate for $i \in\{1, \ldots, M\}$ the Gaussian white noise $Z_{i}$ to the zone $R_{i}$ and $Z_{0}$ to the outside every zone. The new standard Gaussian white noise can be built using:

$$
Z(X)=\prod_{j=1}^{M} \cos \left(\frac{\pi}{2} \alpha_{j}\right) Z_{0}(X)+\sum_{j=1}^{M} \sin \left(\frac{\pi}{2} \alpha_{j}\right)\left(\prod_{k=j+1}^{M} \cos \left(\frac{\pi}{2} \alpha_{j}\right)\right) Z_{j}(X)
$$

We therefore have a model realization $G(Z)$ dependent on the shapes and sizes of the domains $R_{i}$ built by associating one Gaussian white noise to one domain. With this formula, the domain deformation parameters are easier to manipulate. If a grid cell is entirely inside the zone $R_{i}\left(\alpha_{i}=1\right.$ and for all $\left.j \neq i, \alpha_{j}=0\right)$ we have $Z(X)=Z_{i}(X)$.

\section{2.3.2. Combination of the local gradual deformation and the domain deformation}

Both the local gradual deformation method and domain deformation method previously described present some drawbacks. On one hand, it is not easy to define suitable zones for the local gradual deformation method to ensure a potential diminition of the objective function. On the other hand, the domain deformation does not allow the combination of Gaussian white noises inside the zones and therefore limits the possibilities of the optimization process. We propose in this section a new method that generalizes the local gradual deformation by allowing to deform the domains in which the model realizations are combined.

A simple example: One zone and two Gaussian white noises.

Let's start with a simple example where we have two Gaussian white noises $\left(Z_{0}, Z_{1}\right)$ and one zone $R_{1}$ defined in a reservoir model. We propose a new Gaussian white noise that depends on two parameters with $t_{1}$ controling the domain $R_{1}$ and $\rho^{1}$ the gradual deformation.

$$
Z(X)=\cos \left(\pi \alpha_{1}\left(X, t_{1}\right) \rho^{1}\right) Z_{0}(X)+\sin \left(\pi \alpha_{1}\left(\left(X, t_{1}\right) \rho^{1}\right) Z_{1}(X)\right.
$$

where $\alpha_{1}\left(X, t_{1}\right)$ is given by Eq.(8). 
Depending on the position of the grid cell $X$ in the reservoir model and the value of the gradual deformation parameter, we have:

- If $X$ is entirely outside $R_{1}$ then $\alpha_{1}=0$ and $Z(X)=Z_{0}(X)$.

- If $X$ is entirely inside $R_{1}$ then $\alpha_{1}=1$ and

$$
Z(X)=\cos \left(\pi \rho^{1}\right) Z_{0}+\sin \left(\pi \rho^{1}\right) Z_{1}
$$

which corresponds to the gradual deformation technique.

- If $X$ is partially inside $R_{1}, Z(X)$ is a combination of $Z_{0}(X)$ and $Z_{1}(X)$. In this case, both the gradual deformation method and the domain deformation method are applied simultaneously.

- If the gradual deformation is set to a constant $\rho_{1}=\frac{1}{2}$, then $Z(X)=\cos \left(\frac{\pi}{2} \alpha_{1}\right) Z_{0}(X)+\sin \left(\frac{\pi}{2} \alpha_{1}\right) Z_{1}(X)$, which corresponds to the domain deformation method described in section 2.3.1 (Eq.(9)).

One domain and $N+1$ Gaussian white noise.

We can extend the previous case by adding the possibility to combine $N+1$ independent standard Gaussian white noises $\left(Z_{0}, \ldots, Z_{N}\right)$ inside the zone $R_{1} . t_{1}$ still parameterizes the shape of $R_{1}$ and we introduce $N$ gradual deformation parameters $\left(\rho^{1}, \ldots, \rho^{N}\right)$. The new Gaussian white noise proposed is then:

$$
Z(X)=\prod_{j=1}^{N} \cos \left(\pi \alpha_{1} \rho^{j}\right) Z_{0}(X)+\sum_{j=1}^{N} \sin \left(\pi \alpha_{1} \rho^{j}\right)\left(\prod_{k=j+1}^{N} \cos \left(\pi \alpha_{1} \rho^{k}\right)\right) Z_{j}(X)
$$

We have:

- If $X$ is outside $R_{1}$, then $\alpha_{1}=0$ and $Z(X)=Z_{0}(X)$.

- If $X$ is entirely inside $R_{1}$, then $\alpha_{1}=1$ and

$$
Z(X)=\prod_{j=1}^{N} \cos \left(\pi \rho^{j}\right) Z_{0}(X)+\sum_{j=1}^{N} \sin \left(\pi 2 \rho^{j}\right)\left(\prod_{k=j+1}^{N} \cos \left(\pi 2 \rho^{k}\right)\right) Z_{j}(X)
$$

which corresponds to the formula of the gradual deformation method described in Eq.(3).

- If $X$ is partially inside $R_{1}, Z(X)$ is a combination of $Z_{0}(X)$ and $Z_{1}(X)$ created by both the domain deformation and the gradual deformation techniques. 
Extension to the case of $N+1$ model realizations and $M$ domains.

We now extend the method to the general case where we have at our disposal $N+1$ independent Gaussian white noises $\left(Z_{0}, \ldots, Z_{N}\right)$ and $M$ domains $\left(R_{1}, \ldots, R_{M}\right)$ defined on a reservoir model. For each zone $R_{i}$, let $\alpha_{i}$ be defined by Eq.(10) and for each couple "zone"/ "Gaussian white noise" $R_{i} / Z_{j}$, we introduce a gradual deformation parameter $\rho_{i}^{j}$. We propose the generalized formula :

$$
Z(X)=\prod_{j=1}^{N} \cos \left(\pi \sum_{i=1}^{M} \alpha_{i} \rho_{i}^{j}\right) Z_{0}(X)+\sum_{j=1}^{N} \sin \left(\pi \sum_{i=1}^{M} \alpha_{i} \rho_{i}^{j}\right) \prod_{k=j+1}^{N} \cos \left(\pi \sum_{i=1}^{M} \alpha_{i} \rho_{i}^{k}\right) Z_{j}(X)
$$

This is indeed a way to do a gradual deformation of all model realizations in every varying zone but this generalized method introduces too many parameters $(M *(N+1))$. Fortunately, it is possible to reduce the number of parameters just by selecting the "best" model realizations to combine in each zone (setting some of the $\rho_{i}^{j}$ to 0$)$.

11 Let's analyse some cases for a grid cell $X$ :

1. Outside every domains

For each $i, \alpha_{i}=0$. Thus, $Z(X)=Z_{0}(X)$.

2. Suppression of the influence of the realization $l$ in the zone $R_{q}$

Let's set the parameter $\rho_{q}^{l}$ to 0 . If the grid cell $X$ is entirely and uniquely inside $R_{q}, Z(X)$ become:

$$
Z(X)=\prod_{j=1, j \neq l}^{N} \cos \left(\pi \rho_{q}^{j}\right) Z_{0}(X)+\sum_{j=1, j \neq l}^{N} \sin \left(\pi \rho_{q}^{j}\right) \prod_{k=j+1}^{N} \cos \left(\pi \rho_{q}^{j}\right) Z_{j}(X)
$$

The realization $l$ has no more influence in the zone $R_{q}$.

3. Domain deformation of the zone $q$ with only the realization $l$ associated to $R_{q}$

We want to apply $Z_{l}$ inside $R_{q}$. Let's set $\rho_{q}^{l}=\frac{1}{2}$ and for all $j \in\{1, \ldots, l-1, l+1, \ldots, N\}$ the parameters $\rho_{q}^{j}=0$. Then:

- If $X$ is entirely and uniquely inside $R_{q}$ then $Z(X)=Z_{l}(X)$

- If $X$ is only partially inside $R_{q}$, then a combination of $Z_{0}$ and $Z_{l}$ is applied, as for the domain deformation method.

This case corresponds to the domain deformation method applied on the zone $R_{q}$.

4. Gradual deformation on the zone $R_{q}$

If no parameter controlling the shape of $R_{q}$ is introduced then for any grid cell $X, \alpha_{q}$ is constant. This ammounts to perform a local gradual deformation inside the zone $R_{q}$.

This new generalized method offers a great flexibility concerning the definition of different domains and the choice of Gaussian white noises to combine in each one. For example, it is possible to perform a local gradual deformation of several model realizations in one zone, while in parralel applying the domain deformation method to another zone (or any combination of the two methods). 


\section{Initialization of the history matching problem : a patchwork technique}

One of the issues for the history matching problem is its initialization. For the geostatistical local parameterization techniques described in the previous section, choosing relevant domains and model realizations is a key issue to have a good decrease of the objective function. It is therefore necessary to have an efficient way to initialize the history matching problem.

\subsection{Initialization of the history matching problem via patchwork}

By dividing a reservoir model into relatively independent zones, it is possible to initialize the history matching problem by choosing the "best" realizations in each of these zones. The choice of model realizations for a zone can be done by comparing the local value of the objective function and selecting the best one. Let's present the patchwork technique (Reis et al., 2000).

The objective function can be explicited by:

$$
F=\sum_{i=0}^{n} f_{i}\left(x_{1}, \ldots, x_{p}\right)
$$

where $f_{i}$ is the objective function associated to a well $i$ on which we have data to match, $n$ is the number of wells in the reservoir and $\left(x_{1}, \ldots, x_{p}\right)$ are the parameters to be optimized. For a specific zone $k$, if $I$ is the set of wells inside the zone $k$, we call local objective function associated to the zone $k$ the function $g_{k}=\sum_{i \in I} f_{i}$. A patchwork initialization is performed by the following steps:

1. generate $N+1$ model realizations.

2. divide the reservoir into $M$ zones.

3. Name $Z_{0}$ the Gaussian white noise associated to the model realization that presents the lowest objective function value.

4. For each zone $k$ :

- Sort the values of the local objective function. Name $Z_{j}$ the realization such that $g_{k}\left(Z_{j}\right)$ is minimal.

- If $Z_{j} \neq Z_{0}$, apply $Z_{j}$ in the zone $k$ for gradual deformation.

Fig.4 shows an example of a reservoir model divided into 11 relatively independent zones. If the zones are independent from one another, the patchwork initialization is expected to give a better objective function value than $Z_{0}$. However, if the local modification of the model realization in a zone can affect the behavior of the model in the other zones, it is not possible to ensure that the patchwork method will not deteriorate the model. In practice, the independence of different regions is not trivial to be identified and large errors could be committed. It is frequent that a patchwork method increases the value of the objective function. 


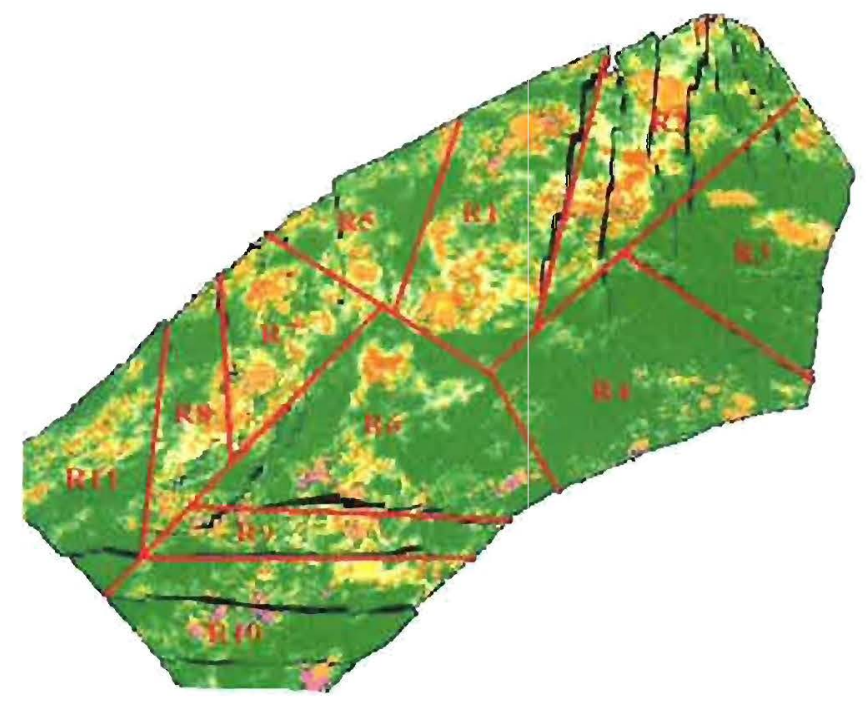

Figure 4: Separation of a reservoir model in 11 independent zones

\subsection{Improved patchwork}

With the new parameterization technique or the domain deformation method, it is more flexible to initialize local zones since their shapes and sizes can be later modified. The patchwork method is improved as follows:

1. Generate $N+1$ model realizations.

2. Name $Z_{0}$ the Gaussian white noise associated to the model realization that presents the lowest objective function value.

3. Group the wells into $M$ subsets $\left(I_{1}, \ldots, I_{M}\right)$. As in the previous section, we call $g_{k}=\sum_{i \in I_{k}} f_{i}$ the local objective function associated to the $k^{\text {th }}$ group of wells. It is possible to choose only one well in each group.

4. For each group of wells $k$ :

- Sort the values of the objective subfunction $g_{k}\left(Z_{j}\right)$. Name $Z_{j}$ the realization such that $g_{k}\left(Z_{j}\right)$ is minimal.

- If $Z_{j} \neq Z_{0}$, define a parametrized zone containing the group of wells $k$ and apply $Z_{j}$ inside. Simple zones defined with one or two parameters are prefered.

5. Start a simple optimization process (for instance, with a single parameter) on the sizes of the domains to have the best possible starting model. In the case of circular zones, we can optimize the sizes of the zones with a single radius parameter. 
Comparing to the patchwork proposed by Reis et al. (2000), parameterized domains are defined in Step 4 and any form of domains can be used instead of a division by regrouping grid cells. Moreover, a simple optimization process is applied in Step 5 to improve the zone sizes. As we will see in examples hereafter,

105 Step 5 allows with a very limited cost (a few reservoir simulations) to get a better initial point.

It is interesting to note that our method leaves a lot of freedom concerning the shape of the zones. Ding \& Roggero (2010) presented several typical domains for history matching. Elliptical domains are suitable for anisotropic permeability correlation media with their two axes proportional to the correlation lengths. They are also adapted to investigate connectivities between a couple of injector/producer with the foci of the ellipse at the wells. The simplest domain shape is the circle with its center fixed at a well and a radius $r$ to be optimized. In the following, we will illustrate some examples using radial domains parameterized with their radius.

\section{Numerical results}

In this section, we tested our new initialization and parameterization techniques on three cases. The first one is a simple synthetic reservoir model, the second one is the reservoir model PUNQ (Barker et al., 2000) and the last one is an adapted Brugge model (Chen et al., 2010, Peters et al., 2010, 2012). In the simple synthetic case and the Brugge case, as the correlation lengths are nearly isotropic, we worked with circular zones. Anisotropic correlation lengths are present in the PUNQ reservoir, but each layer has a different anisotropy and different axis: their azimuths are respectively 30, 0, 45,-30 and 60 degrees from the principal axis. As it is difficult to favor a particular layer over the others and we don't want to introduce one new parameter per layer around each well, we also chose to work with circular domains for this test. We will briefly describe the studied cases before showing the numerical results.

\subsection{Description of the three studied cases}

Simple synthetic case. The dimensions of the field are $2500 \mathrm{~m}$ in the $x$ direction, $2500 \mathrm{~m}$ in the $y$ direction and $10 \mathrm{~m}$ in the $z$ direction. It is discretized uniformly by $50 \times 50 \times 1$ grid cells of $50 \mathrm{~m}$ in the $x$ direction, $50 \mathrm{~m}$ in the $y$ direction and $10 \mathrm{~m}$ in the $z$ direction. There are 25 vertical wells containing 12 water injectors and 13 producers (Fig.5). The reservoir is heterogeous and the correlation length is $150 \mathrm{~m}$ in the $x$ and $y$ directions. The initial reservoir pressure is $250 \mathrm{bars}$ and a production data history of 4000 days is known. Well bottom hole pressures are imposed with 320 bars on the injectors and 180 bars on the producers. We consider for our objective function to be minimized the water rate on the 12 injectors as well as the oil rate and the water-cut on the 13 producers. 


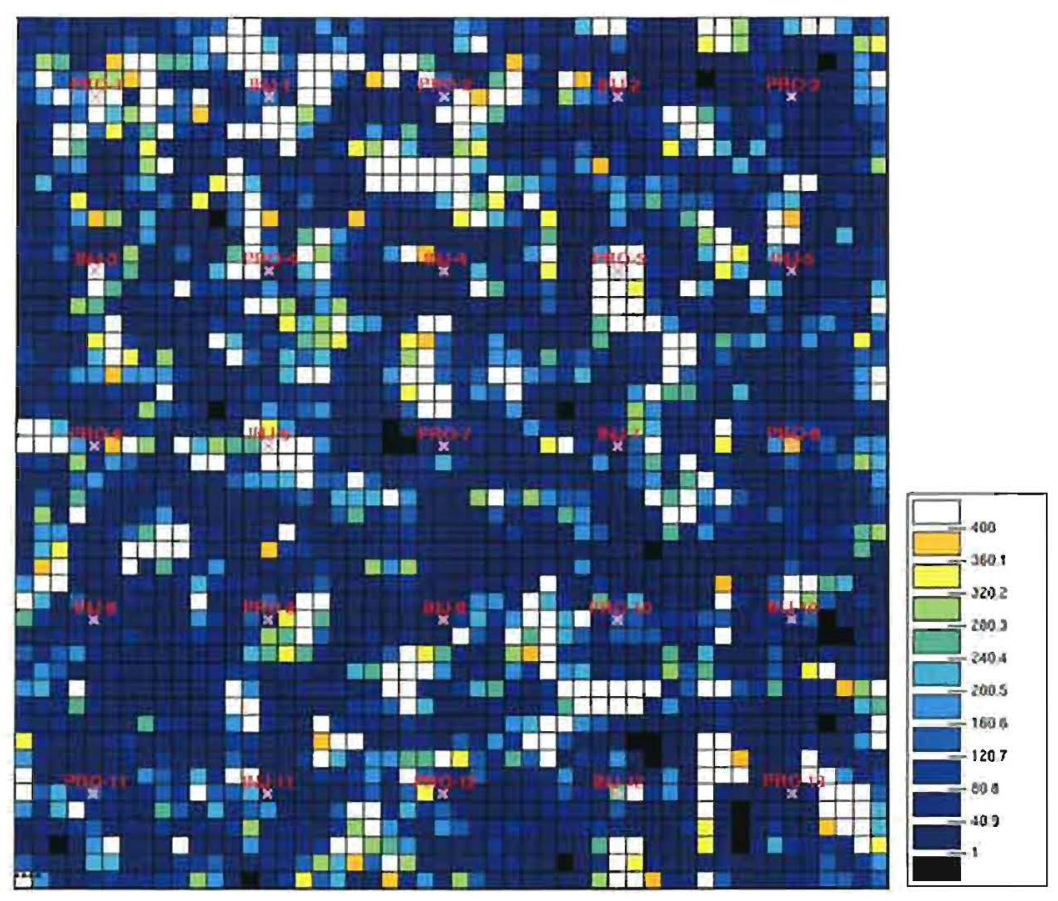

Figure 5: Permeability map of the synthetic oil field

PUNQ. The reservoir PUNQ is a synthetic case which results from a field study conducted by the industrial partners of the project PUNQ (Barker et al., 2000). The model is constituted of 19x28x5 grid cells of which 1761 are active. Six producing and seven injection wells are present. Fig.6 shows the permeabilty at the top layer of the reservoir for a realization of the model. Porosity and permeability maps have been generated with the geostatistical method FFTMA (Le Ravalec et al., 2000). We have at our disposal a production history of 12 years. We use for our objective function the parameters BHFP (Bottom Hole Flowing Pressure), WC (Water CUT) and GOR (Gas Oil Ratio) for the producing wells and BHFP on the injecting wells.

Brugge. Brugge is a synthetic reservoir model built for the 2008 Applied Technology Workshop (Peters et al., 2010, 2012). The original model has 20 millions grid cells with average sizes of $50 \mathrm{~m} \times 50 \mathrm{~m} \times 0.25 \mathrm{~m}$. We worked with an upscaled model of 60 thousands grid cells. 30 wells are present on the reservoir, 10 are injecting wells and 20 are producing wells. In this work, we generated our history data by creating reference porosity and permeability maps with the geostatistical method FFTMA (Le Ravalec et al., 2000), and we compare different optimization techniques to match these history data. Figure 7 shows the permeability map of the first layer of the reservoir for the reference realization of the model. The production has a history of 10 years. We use for our objective function the parameters BHFP (Bottom Hole Flowing Pressure) and WC (Water Cut) for the producing wells and BHFP on the injecting wells. 


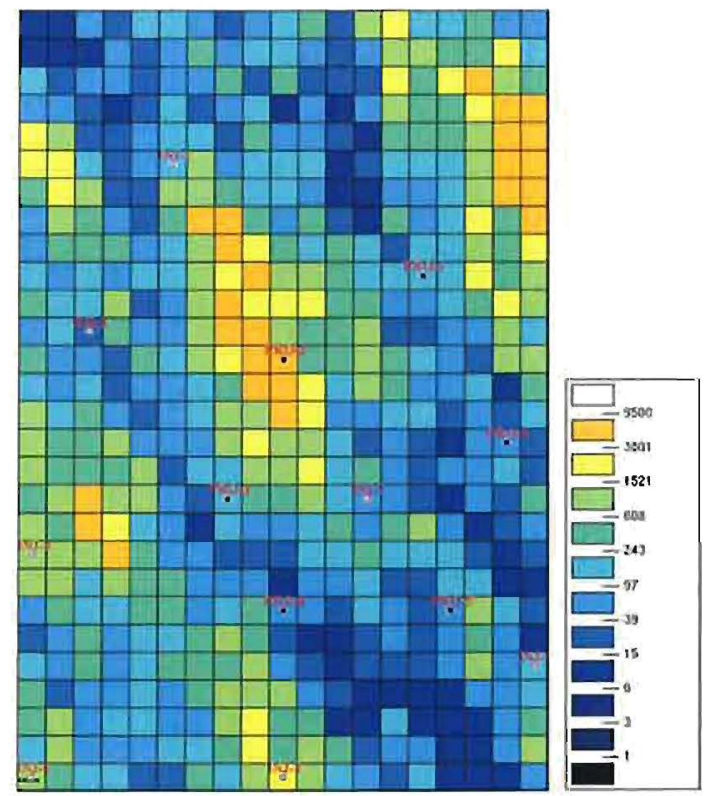

Figure 6: Permeability and well position of the top layer of the PUNQ reservoir

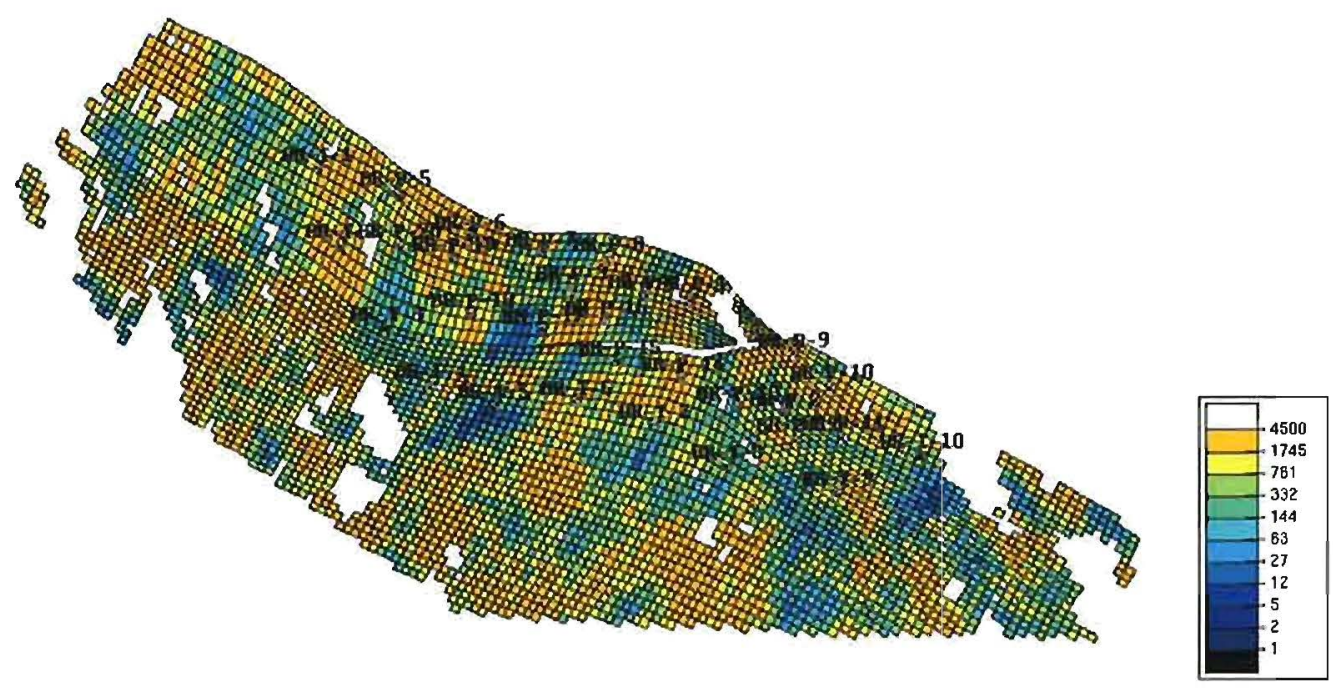

Figure 7: Permeabilty and well positions on the Brugge reservoir

\subsection{Model initialization}

Simple synthetic case. We generated 5 sets of 25 random realizations to apply the patchwork method with radial domains around the wells. Fig.8 illustrates the new patchwork method: the zones created are the circles around the wells. In this figure 16 zones were created, the problem is therefore parameterized with 16 gradual deformation parameter and 16 domain deformation parameters (radius of the domains).

Fig.9 shows the values of the objective function in each of our 5 cases for the best realization and the 


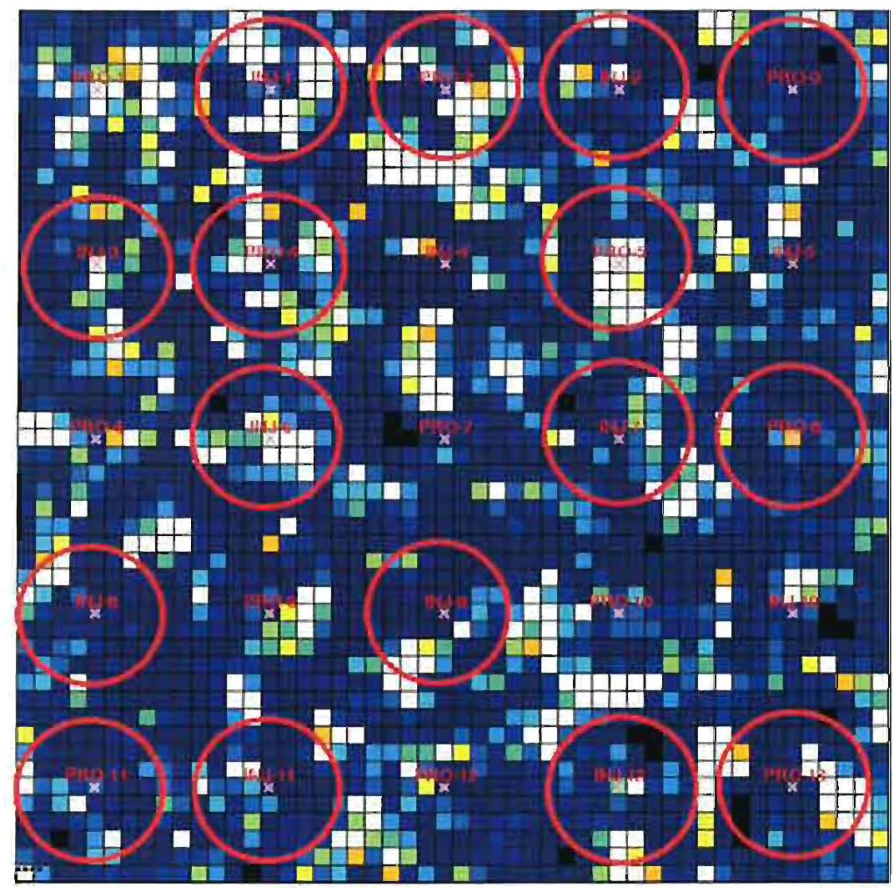

Figure 8: Initialization for the new parameterization method via modified patchwork on a synthetic reservoir model

235 realization given by the patchwork technique with fixed a radius $r=300 m$ on all the domains. For the first 4 cases, the patchwork method is beneficial without any further evaluation of the objective function. However, for the last case, the objective function has been increased by the patchwork technique. In consequence, we

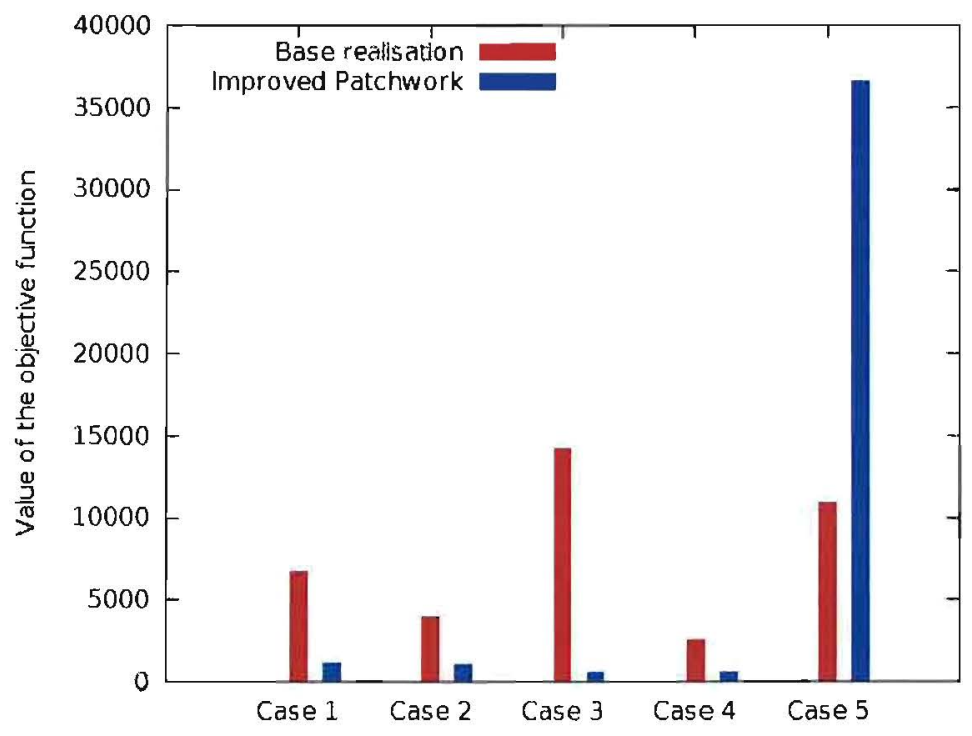

Figure 9: Value of the objective function for the best realization (in red) and for the realization obtained by patchwork with $r=300 m$ (in blue) in 5 cases 
drew for that case in Fig.10 the value of the objective function after patchwork as a function of the domains radius. It is observed that the objective function is deteriorated with the radius $r=300 \mathrm{~m}$. However, there is a clear minimum on this curve that is inferior to the value of the "best" realization (horizontal bar in blue). A gradient based optimization method can find this minimum in 5 evaluations of the objective function. Radial domains give a better initial model with a radius of $r=206 \mathrm{~m}$ for the optimization proccess.

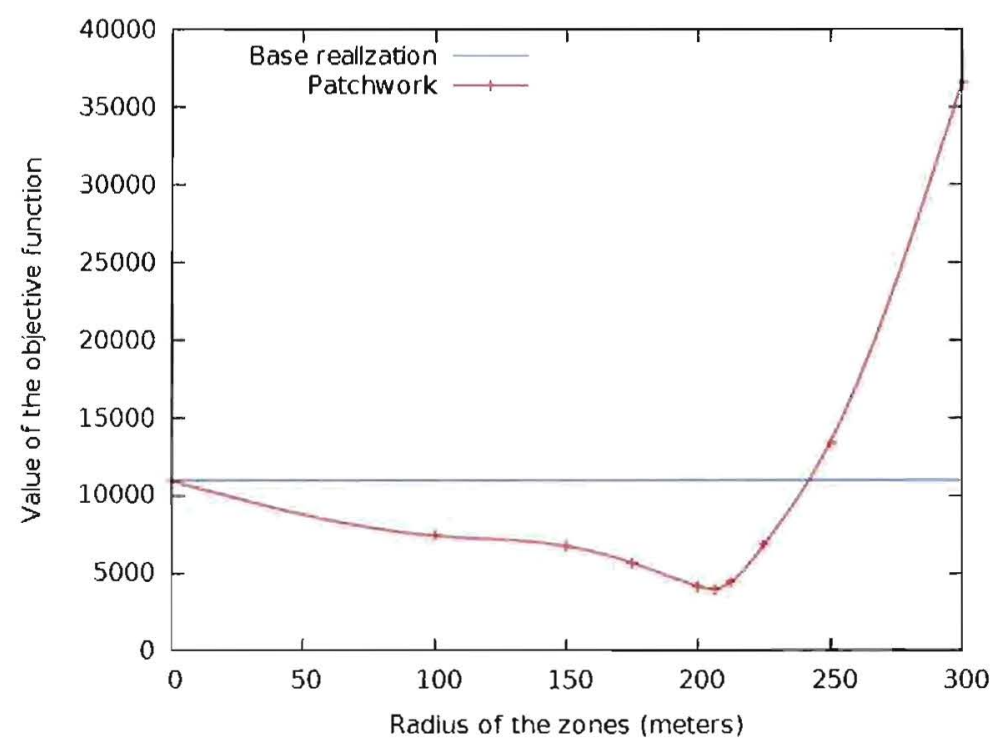

Figure 10: Objective function after patchwork in respect with the radius of the zones

PUNQ. As for the previous example, we generated 11 random model realizations for the PUNQ case. The new patchwork technique created 9 effective zones because the local objective functions associated to the four other wells are the minimum on the base case (the "best" random realization). Fig.11 shows the value of the objective function after patchwork with respect to the radius of the zones. The minimum is clearly inferior to the value of the objective function given by the "best" realization. We found this minimum with 7 evaluations of the objective function through a gradient-based optimization method. The objective function goes from 649.9 to 502.0 with the new patchwork approach showing a relative gain of $22 \%$.

To compare with the initial point given by the classical patchwork technique (Reis et al., 2000), we divided the grid into zones delimited by grid cells as shown in Fig.12. In this example, each zone contains one and only one well. Using this domain division, the objective function increases from 649.9 for the best global realization to 851.1. Unfortunately, the classical patchwork method is detrimental to the objective function but we have no mean to improve it. 


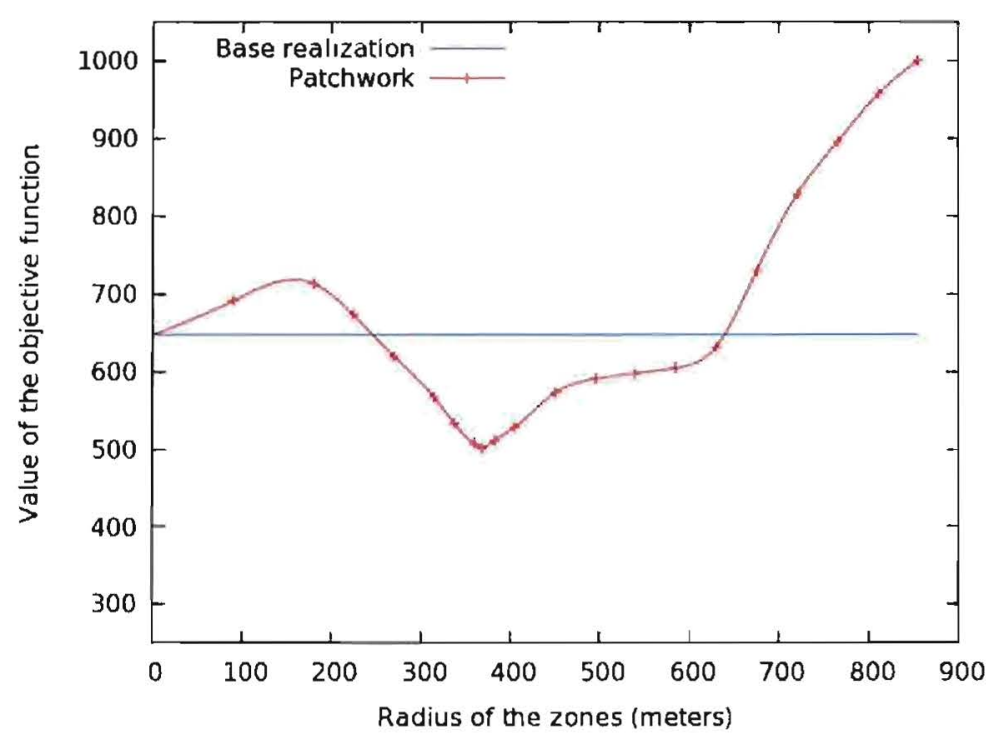

Figure 11: Value of the objective function after patchwork in respect with the radius of the zones for the PUNQ case

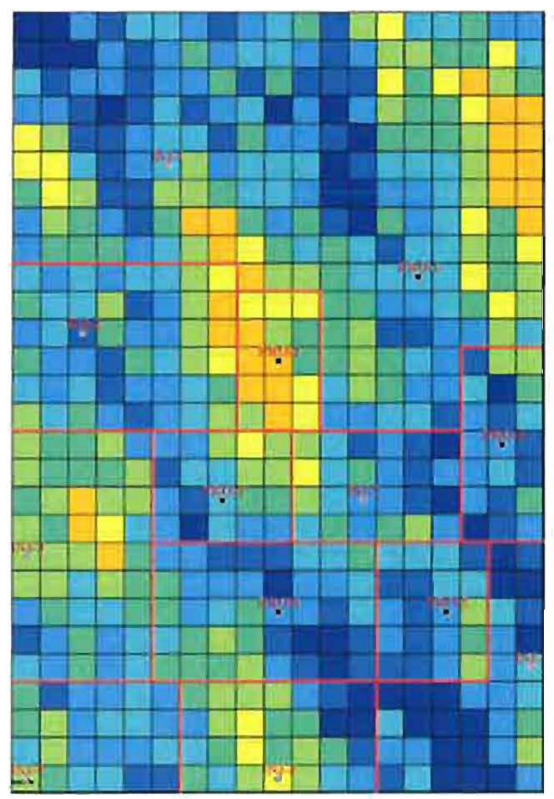

Figure 12: An engineering division of the PUNQ reservoir into zones

It is interesting to note that even in the case where the objective function cannot be improved, the new patchwork method is not detrimental. In fact, when all the radius are reduced to zero, we obtain the "best" realization among all the random realizations.

Brugge. For this case, 19 random model realizations were generated. The new patchwork technique created

7 zones around the wells $P-10, P-13, P-15, P-16, P-19, P-2$ and $P-5$. Fig.13 shows the value 
of the objective function after patchwork with respect to the radius of the zones. Again, the minimum is inferior to the value of the objective function given by the best realization. We could find this minimum in

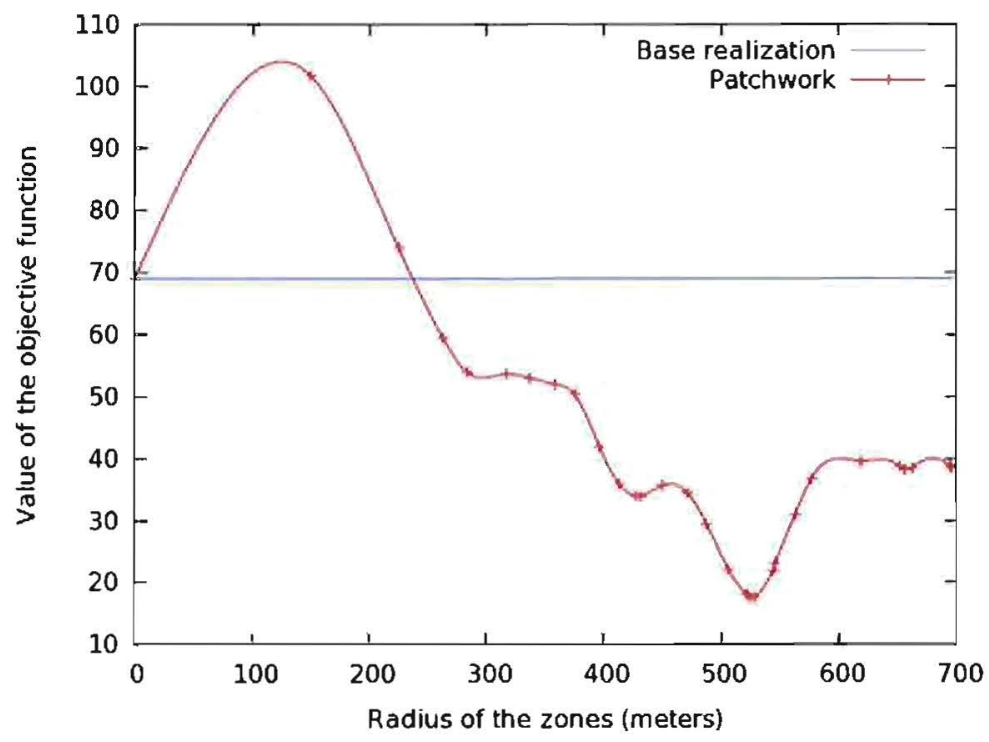

Figure 13: Value of the objective function after patchwork in respect with the radius of the zones for the Brugge case

only 6 objective function evaluations using a gradient based optimization method for one radius parameter. The objective function goes from 68.9 to 17.5 with the patchwork approach, showing a relative gain of over $20574 \%$.

As for the PUNQ case, we compared our results to the one given by the classical patchwork method where we created zones delimited by grid cells around the wells (Fig.14). Using this reservoir division, the patchwork technique gives an objective function value of 45.2 . In this case, the method improves the objective function but is still far from the results given by our method.

\subsection{Numerical results of the generalized gradual deformation method}

We use the model obtained by patchwork in the previous session as the starting point for the history matching. In all tests, a gradual deformation parameter is introduced on each zone to combine the local best realization with the overall best random realization, and a domain parmeter is also introduced to control the radius of the zones.

Simple synthetic case. The generalized gradual deformation method is compared to the local gradual deformation. A gradient based optimization approach has been applied in the optimization process. Fig.15 shows the decrease of the objective function with respect to the number of evaluations for the five cases.

In each case, the decrease rate of the objective function with respect to the number of reservoir simulations obtained with the generalized gradual deformation method is similar to the one obtained with the classical 


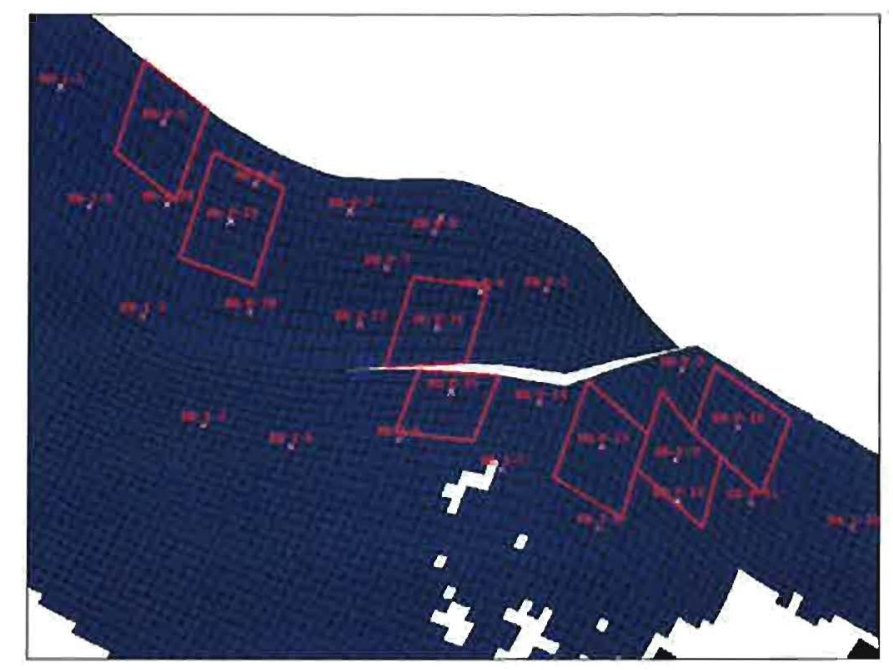

Figure 14: An engineering division of the Brugge reservoir into zones

280 local gradual deformation method, but the generalized gradual deformation method gives a better optimal result at the end (respectively 5\%,11\%,20\%,47\% and 27\% relative gain on the gradual deformation method). Even with an increased number of parameters, this new parameterization method gives a better result in a similar number of objective function evaluations. These results show the importance of taking into account the size of the local zones for history matching for the local gradual deformation. 

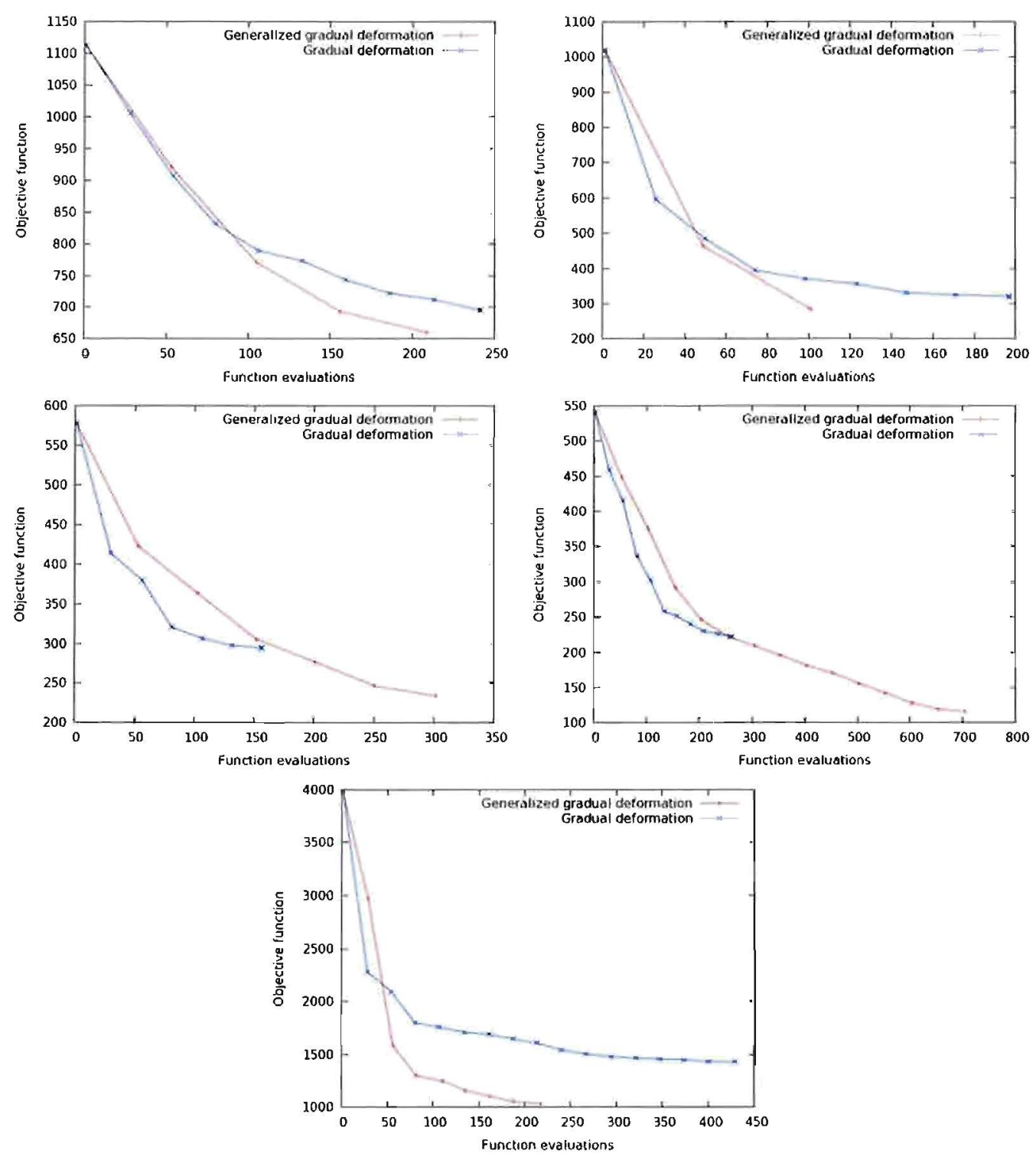

Figure 15: Comparison of the generalized gradual deformation and the gradual deformation techniques on 5 instances of the synthetic case.

${ }_{285}$ PUNQ. We work in this test with 9 zones (see section 4.2). We start by comparing the performances of our generalized method and the local gradual deformation method on an engineering defined zones delimited by grid cells (Fig.12). Only the definition of the zones differs between the two methods : we consider the same number of zones and combine the same model realizations inside the zones. Results are shown on Fig.16.

The optimization starts with the base realization (best realization among the 10 random ones initially generated). The first step on the generalized gradual deformation curve corresponds to the initialization technique (section 3) and illustrates that we needed 7 evaluations to find a better initial point by optimizing 


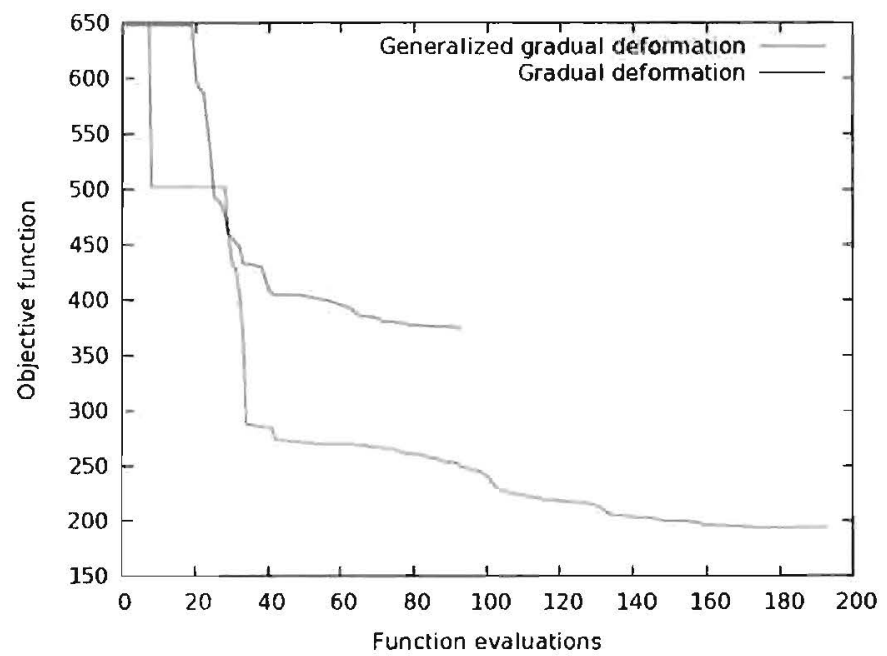

Figure 16: Optimization of the objective function using the local gradual deformation and the generalized gradual deformation on the PUNQ case

the size of the zones. The generalized gradual deformation method gives a far better result than the classical gradual deformation technique (relative gain of over $48 \%$ ).

To further illustrate our method, we also compared three variant parameterization techniques from the best initial point obtained with the improved patchwork: the generalized gradual deformation method (9 radius parameters as we have 9 zones), the generalized gradual deformation method with one radius parameter for all the zones and the local gradual deformation method where the radius are unchanged. For this case, a derivative free optimizer (Langouët, 2012) was chosen to minimize our objective function. Results are presented in Fig17.

We can see in this figure that all the three methods give a similar decrease rate for the objective function. However, the methods that allow the sizes of the zones to vary give better final results (about $15 \%$ relative gain). Moreover, it seems sufficient to introduce only one parameter to controle the sizes of all the zones in this example. These simulations also show that taking into account the sizes of the zones can add a great flexibility to the parameterization technique without increasing greatly the cost of the history matching process. 


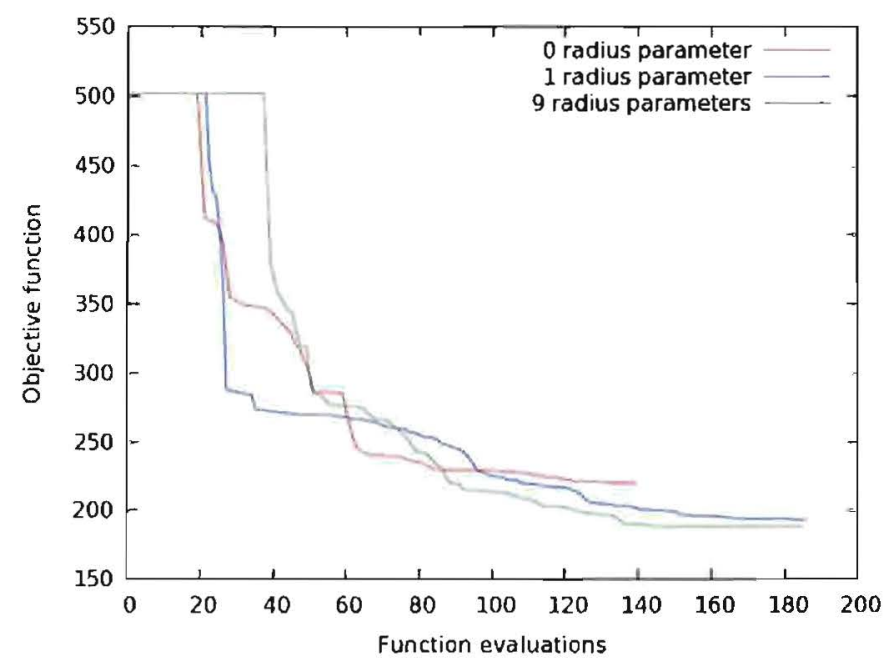

Figure 17: Optimization of the objective function for the 3 parameterization techniques on the PUNQ case

Brugge. We work in this test with 7 zones (see section 4.2). We performed two history matching, one using the local gradual deformation method on the zones delimited by grid cells (Fig.14) and one using our parameterization method. We show in Fig.18 the results we obtained.

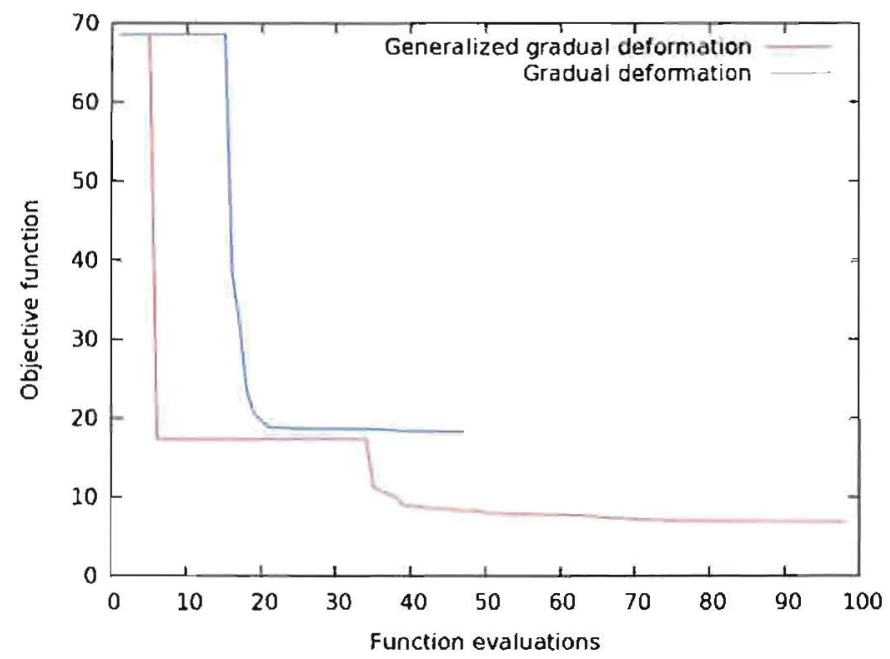

Figure 18: Optimization of the objective function using the local gradual deformation and the generalized gradual deformation on the Brugge case

In this case, the initialization method alone gives a better model realization than the local gradual deformation method. Our method presents a final relative gain of $60 \%$. It is quite evident that a good definition of the zones is crucial to have a fast decrease of the objective function for this test. 


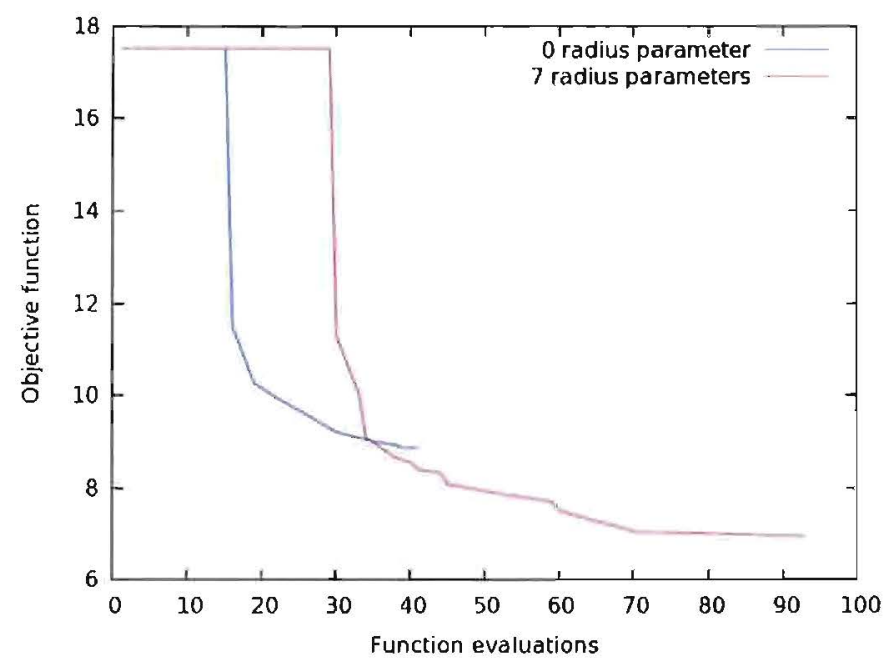

Figure 19: Optimization of the objective function for two variant parameterization techniques on the Brugge case

We also compared two variant parameterization techniques, one with 7 radius parameters and one without radius, from the best point obtained with the improved patchwork. We optimized the history matching objective function using the SQA (Langouët, 2012) optimizer. Results are presented on Fig 19. As in the previous tests, we were able to get a better model realization with a radius parameter for each zone (relative gain of $21 \%$ ). Taking the size of the zones into account allows for a greater decrease of the objective function.

\section{Conclusion}

We presented in this paper a new parameterization method that remedies one of the biggest flaws of the local gradual deformation technique in history matching. The quality of the local gradual deformation method depends strongly on the initial set of local domains. To overcome this shortcoming, a generalized formulation is proposed, which modifies the geostatistical realization with the gradual deformation method inside local domains while the shapes and sizes of these domains are also changed by the domain deformation technique. This method allows the history matching optimization algorithm to dynamically modify the sizes of the domains and therefore reduces the dependence of the optimal realization on an initial expert guess for local domains selections. We successfully showed better results with our generalized gradual deformation method than those with the standard gradual deformation technique on three history matching problems. Therefore we are confident that this new method is a good generalization of the gradual deformation technique and may allow good history matching on a greater number of cases. 


\section{References}

Al-akhdar, S., Ding, D. Y., Dambrine, M., \& Jourdan, A. (2012). An integrated parameterization and optimization methodology for assisted history matching: Application to libyan field case. In SPE North Africa Technical Conference and Exhibition, Cairo, Egypt. SPE 150716.

33 Barker, J., Cuypers, M., \& Holden, L. (2000). Quantifying uncertainty in production forecasts: Another look at the punq-s3 problem. SPE Journal, 6(4), 433.441.

Chen, C., Wang, Y., Li, G., \& Reynolds, A. (2010). Closed-loop reservoir management on the brugge test case. Computational Geosciences, 14(4), 691-703.

Ding, D. Y., \& Roggero, F. (2010). History matching geostatistical model realizations using a geometrical domain based parameterization technique. Mathematical Geosciences, 42(4), 413-432.

Gervais, V., Gautier, Y., Le Ravalec, M., \& Roggero, F. (2007). History matching using local gradual deformation. SPE EUROPEC/EAGE Conference and Exhibition, .

Hoffman, B., \& Caers, J. (2003). Regional probability perturbations for history matching. SPE Annual Technical Conference and Exhibition, .

Hu, L. (2002). Combination of dependant realizations within the gradual deformation method. Mathematical Geology, 34(8), 953-963.

Langouët, H. (2012). Optimisation sans dérivées sous contraintes. Ph.D. thesis Université de Nice-Sophia Antipolis.

Le Ravalec, M., Noetinger, B., \& Hu, L. (2000). The fft moving average (fft-ma) generator: An efficient numerical method for generating and conditioning gaussian simulations. Mathematical Geology, 32(6), 701-723.

Marsily, G., Lavedan, C., Boucher, M., \& Fasanino, G. (1984). Interpretation of interference tests in a well field using geostatistical techniques to fit the permeability distribution in a reservoir model. Geostatistics for Natural Resources Characterization, 122(2), 831-849.

Peters, E., Arts, R., Brouwer, G., Geel, C., Cullick, S., Lorentzen, R., Chen, Y., Dunlop, K., Fossepoel, F., Xu, R., Sarma, P., Alhutali, A., \& Reynolds, A. (2010). Results of the brugge benchmark study for flooding optimization and history matching. SPE Reservoir Simulation Symposium, the Woodlands, Texas, USA, .

Peters, E., Chen, Y., Leeuwenburgh, O., \& Oliver, D. (2012). Extended brugge benchmark case for history matching and water flooding optimization. Computers \& Geosciences, 50, 16-24. 
Reis, L., Hu, L., de G. Marsily, \& Eschard, R. (2000). Production data integration using a gradual deformation approach : application to an oil field. SPE Annual Technical Conference and Exhibition, .

Roggero, F., Ding, D. Y., Berthet, P., Lerat, O., Cap, J., \& Schreiber, P. (2007). Matching of production history and $4 \mathrm{~d}$ seismic data - application to the girassol field, offshore angola. SPE 109929, Proc. SPE ATCEE, Anaheim, California, .

Roggero, F., \& Hu, L.-Y. (1998). Gradual deformation of continuous geostatistical models for history matching. SPE Annual Technical Conference and Exhibition, . 